\title{
HACIA EL AULA VIRTUAL: ACTIVIDADES DE ENSEÑANZA Y APRENDIZAJE EN LA RED
}

\author{
Elena Barbera y Antoni Badia \\ Universitat Oberta de Catalunya, España
}

\section{CONCEPTO DE AULA VIRTUAL ESCOLAR}

La situación de los ordenadores en un centro escolar y el rol que desempeñan en él puede resultar, a día de hoy, un tanto polémica. Pensamos que la controversia no procede nuclearmente de los mismos centros a los que se les acumula un sinfín de responsabilidades, sino de las propias demandas sociales e institucionales que yuxtaponen uno detrás de otro los trabajos que debe desarrollar un centro escolar. Naturalmente, los centros se ven en la necesidad de ordenar, integrar y dar respuesta a todas estas demandas nuevas y crecientes, situación que no es siempre fácil si la leemos acompañada de una cierta falta de recursos personales, materiales y formativos. Con este panorama no queremos más que apuntar la realidad escolar que por lo que respecta a la tecnología más puntera se mueve entre el binomio que determina, por un lado, el lujo y, por otro, la necesidad de tener un ordenador en la clase. Más allá de discutir en detalle si ese es el lugar que ha de ocupar en un centro, creemos que cualquier profesor puede estar de acuerdo en tener un aula bien dotada y que ello incluya también un ordenador en alguna parte del espacio del que él es educativamente responsable. El ordenador es un medio que como lo es la televisión, el vídeo, la prensa, la radio, las diapositivas, etc. que tienen como finalidad ayudarle en su tarea docente. Ahora bien, el uso del ordenador es múltiple y singular, por tanto, aunque comparte las características de todos los medios de utilización educativo tiene particularidades que lo hacen único.

Nuestra opción en este escrito y, por supuesto en la práctica, es optar por la concepción de clases en las que se integre un ordenador con conexión a la red y se organice un plan de trabajo sistemático a diferentes niveles según los objetivos que se marquen para los alumnos. Más concretamente, lo ideal sería contar con un aula virtual dentro de la clase que ofreciera la posibilidad de preparar un conjunto de actividades útiles para el profesor y para los alumnos e integradas en el propio currículum escolar y que no fueran de tipo anecdótico o se realizaran de manera asistemática o a las que sólo accedieran unos cuantos. Cuando nos referimos a una aula virtual estamos pensando en un conjunto de actividades con un alto ingrediente comunicativo que de otro modo, es decir, sin la existencia del ordenador conectado a la red, no tendrían el sentido que adquiere en el contexto auténtico que proporciona la tecnología.

Por tanto, entendemos que un aula virtual se puede situar dentro de la misma clase y, aunque podría también funcionar de manera autónoma como sucede en la educación superior, puede cubrir una serie de necesidades educativas nada despreciables realizadas de manera que se libere al profesor y al alumno de la coincidencia temporal e incluso espacial. Esta es la gran diferencia entre un aula virtual y una presencial junto con las posibilidades educativas que ofrece de flexibilización de los itinerarios personales y 
el desarrollo de capacidades de tipo exploratorio, procesual y de visualización. En resumen, estamos hablando de la posibilidad de diversificar y adaptar la oferta y la ayuda educativa a diferentes niveles y momentos sin que el profesor tenga que estar presente de una manera dominante y homogénea para todos los alumnos. Por tanto, nos movemos en un lapso de tiempo y en un espacio en el que el alumno trabaja de manera autónoma pero a la vez necesita un motivo y una guía para realizar sus actividades y darles el sentido educativo que se ha considerado.

Existen dos grandes ejes de confección de acciones educativas virtuales: 1. el primero, presidido por el lugar en el que sucede la acción educativa virtual en relación a la clase convencional y 2. el segundo, referido al uso que se hace del medio tecnológico en el desarrollo de la acción virtual. Ambos ejes confieren singularidad a las diferentes propuestas de actividades en un aula virtual.

\subsection{En el primer eje, diferenciamos entre:}

a) acciones desarrolladas en la propia clase pero que tienen una naturaleza virtual, distribuida en el espacio y el tiempo con ayuda de la tecnología; es decir, en las que se introducen algunos elementos de desarrollo y de apoyo tecnológico al aprendizaje realizado en la misma clase. Por ejemplo, es el caso de las actividades de ampliación o de refuerzo como complementarias al trabajo desarrollado en las áreas curriculares, como puede ser la confección de una carta electrónica dirigida a un estamento real para hacer una consulta con la intención y la posibilidad de obtener una respuesta para tomar una decisión en la clase; o el desarrollo de un proyecto virtual de tipo colaborativo entre alumnos de diferentes centros escolares o de culturas diferentes que puede estar supervisado por el profesor en los momentos que él mismo establezca.

b)Otro tipo de acciones son las que hacen referencia a acciones realizadas fuera de clase pero que tienen una relación directa con el trabajo escolar. Nos referimos a la inclusión de mecanismos de soporte a la comunicación y al estudio realizado por parte de los alumnos en otro lugar que no es la escuela, como puede ser, por ejemplo, el trabajo llevado a cabo en casa como los deberes o la relación que puede existir entre la familia y la escuela que puede estar facilitada por el uso de la tecnología telemática.

1.2 En el segundo eje que se refiere al uso que se hace del medio tecnológico en el desarrollo de la actividad en un aula virtual identificamos al menos cuatro grandes usos:

- a)uso de programas de ordenador como herramientas, como por ejemplo, los instrumentos de gestión de la información como pueden ser un procesador de textos, una hoja de cálculo o un programa de edición de documentos digitales;

- b)uso de medios, programas o materiales de acceso y comunicación del contenido curricular, léase, CD-Rom, Internet o programas que configuran entornos de exploración e indagación;

- c) uso de programas o materiales como instrumento de soporte a la construcción de conocimiento específico de un área curricular, como por ejemplo, los tutoriales o las simulaciones;

- d)uso de herramientas de comunicación entre los participantes, tanto entre los profesores como entre los alumnos entre ellos y entre profesores y alumnos, como puede ser el correo electrónico, las discusiones virtuales o los grupos cooperativos virtuales.

Un aspecto importante es entrelazar las actividades presenciales con las virtuales, hechas en casa $o$ en clase, de modo que formen parte de un mismo tejido y de unos mismos objetivos de manera que se 
utilicen con normalidad unas y otras aunque para ello se precisa un análisis previo de ubicación conceptual del aula virtual en cada uno de los centros. Es posible que un centro por las características de las familias que acoge decida no dar soporte obligatorio al trabajo realizado en casa, pero sí que puede ofrecerles la posibilidad de conexión para realizar tareas de clase en horario extraescolar como sucede con algunas bibliotecas escolares.

Para tener un aula virtual no es necesaria una tecnología muy complicada. Aunque cuando nos referimos a un aula virtual ésta debería estar tan o más organizada que la clase presencial con todos sus recursos disponibles -ver ejemplo adjunto-, para empezar a utilizar el concepto de aula virtual el profesor puede emplear el ordenador de manera que disponga organizadamente de los recursos que le ofrece la red; más adelante, por ejemplo, se puede confeccionar su propia página web en la que distribuya y relacione los recursos que va a utilizar -bases de datos, direcciones electrónicas, foros, debates, trabajo en grupo, librerías virtuales, actividades de aprendizaje y autoevaluación, etc.-. En sentido propio, el aula virtual es la integración organizada de muchos recursos digitales de texto, imagen, sonido y animación; existe software asequible que propone y facilita su personalización, como la aplicaciones webCT, FirstClass o similares. De todos modos, antes de pensar en las actividades de aprendizaje que se realizarán virtualmente para confeccionar un entorno de estas características se deberán tener en consideración un conjunto de factores (Passey, 2000): a) la tecnología disponible, dado que ello mediatiza las posibilidades de desarrollo educativo; b) la tipología de actividades que queremos incluir con el uso de la tecnología disponible; c) las características del acceso al aula y los diferentes agentes que acceden (saber desde dónde, cuándo, quién, duración, etc.); d) los motivos y las necesidades del uso de la tecnología: si son más comunicativos, exploratorios, de apoyo, etc.; e) el enfoque, la naturaleza y el tipo de adquisición del conocimiento en relación a las preferencias de aprendizaje; y, f) el modo como puede organizarse el aprendizaje en función del tipo de interacción deseado y la manera como se mantendrá ese nivel de interacción educativa.

Ejemplo de organización de aula virtual

\begin{tabular}{|c|c|}
\hline $\begin{array}{c}\text { Funciones: Escribir/ responder/ consultar } \\
\bullet \quad \text { Actividades y cronograma } \\
\bullet \quad \text { Autoevaluación } \\
\text { - } \text { Debate } \\
\text { - } \quad \text { Webuestas de la asignatura }\end{array}$ & $\begin{array}{l}\text { - Biblioteca digital: } \\
\text { - BBDD: } \\
\text { - Links de interés }\end{array}$ \\
\hline
\end{tabular}

\section{FUNCIONES EDUCATIVAS DE UN AULA VIRTUAL, OBJETIVOS Y MODALIDADES DE ORGANIZACIÓN DE LAS ACTIVIDADES}

La emergencia de aulas virtuales en el seno escolar se debe valorar no tanto como un aporte al que nos vemos abocados debido a la actual "moda virtualizadora", sino en relación a las funciones sociales y educativas que desempeña su utilización por parte de los alumnos.

Las funciones de un aula virtual son el referente que evalúa la conveniencia de su inclusión en las escuelas y aunque son de diversa naturaleza son funciones relacionadas entre ellas. A parte de enumerarlas y explicarlas brevemente para dar sentido a las aulas que queremos incorporar, estas funciones las entendemos directamente ligadas a objetivos educativos concretos relacionados con la integración de las TIC en educación que se traducen en unas tipologías de actividades de enseñanza y aprendizaje a las que también queremos dar nombre. Por tanto, no hablaremos de funciones aisladas $u$ objetivos inconexos, sino que los relacionaremos y les atribuiremos una forma posible de adquirirlos virtualmente aumentando así su propio sentido. Esta conexión que en la mayoría de casos queda cuanto 
menos implícita e incluso desdibujada nos parece de irrenunciable declaración en el marco de una enseñanza y un aprendizaje de tipo virtual.

En el cuadro siguiente se resume la propuesta de estas relaciones y se aportan los elementos básicos para que el profesor pueda decidir sobre la puesta en práctica de elementos metodológicos que se explicarán con mayor detalle en el apartado siguiente:

Tabla 1

\begin{tabular}{|c|c|c|}
\hline $\begin{array}{c}\text { FUNCIONES } \\
\text { ¿A qué responde la } \\
\text { incorporación virtual? }\end{array}$ & $\begin{array}{c}\text { OBJETIVOS } \\
\text { ¿Qué quiero que el alumno aprenda? }\end{array}$ & $\begin{array}{c}\text { ACTIVIDAD } \\
\text { ¿Qué modalidad } \\
\text { organizativa de la tarea } \\
\text { parece más } \\
\text { conveniente? }\end{array}$ \\
\hline 1. Socializadora & $\begin{array}{l}\text { Colaborar en la inserción progresiva del alumno } \\
\text { en la sociedad de la información y la } \\
\text { comunicación y en el desarrollo de la propia } \\
\text { cultura. }\end{array}$ & $\begin{array}{l}\text { Comunidades virtuales de } \\
\text { aprendizaje }\end{array}$ \\
\hline 2. Responsabilizadora & $\begin{array}{l}\text { Comprometerse e implicarse en el propio } \\
\text { aprendizaje al asumir el reto de aprender } \\
\text { mediante un nuevo medio. }\end{array}$ & Contratos virtuales \\
\hline 3. Informativa & $\begin{array}{l}\text { Consultar diversidad de informaciones } \\
\text { provenientes de fuentes también diversas. }\end{array}$ & Internet \\
\hline 4. Comunicativa & $\begin{array}{l}\text { Expresar los propios conocimientos, experiencias } \\
\text { y opiniones en un contexto comunicativo real. }\end{array}$ & Discusiones virtuales \\
\hline $\begin{array}{l}\text { 5. Formativa } \\
\text { Formadora }\end{array}$ & $\begin{array}{l}\text { Construir conocimiento compartido con el } \\
\text { profesor y otros compañeros con su ayuda. }\end{array}$ & Trabajo colaborativo \\
\hline 6. Motivadora & $\begin{array}{l}\text { Ampliar los conocimientos personales siguiendo } \\
\text { itinerarios personales y mediante la exploración } \\
\text { libre } u \text { orientada. }\end{array}$ & Edición web \\
\hline 7. Evaluadora & $\begin{array}{l}\text { Plasmar el aprendizaje realizado y argumentar } \\
\text { los procesos de comprensión de los contenidos. }\end{array}$ & $\begin{array}{l}\text { Preguntas de corrección } \\
\text { automática }\end{array}$ \\
\hline 8. Organizadora & $\begin{array}{l}\text { Ordenar la propia manera de proceder en el } \\
\text { proceso de aprendizaje. }\end{array}$ & BBDD personales \\
\hline 9. Analítica & $\begin{array}{l}\text { Indagar mediante la observación y comparación } \\
\text { e datos obtenidos y realizarse preguntas al } \\
\text { respecto. }\end{array}$ & Proyectos electrónicos \\
\hline 10. Innovadora & $\begin{array}{l}\text { Integrar diferentes medios tecnológicos para } \\
\text { obtener un resultado funcional. }\end{array}$ & $\begin{array}{l}\text { Material multimedia } \\
\text { presentaciones ppt }\end{array}$ \\
\hline 11. Investigadora & $\begin{array}{l}\text { Probar el método científico en relación a } \\
\text { pequeños estudios personales. }\end{array}$ & Investigaciones virtuales \\
\hline
\end{tabular}

\section{DIMENSIONES Y ACTIVIDADES DE ENSEÑANZA Y APRENDIZAJE}

Seguidamente vamos a caracterizar un conjunto de actividades de enseñanza y aprendizaje desarrollados parcial o totalmente en aulas virtuales. En cada caso se van a indicar dos dimensiones:

Tabla 2

Dimensiones a tener en cuenta en el diseño, planificación y desarrollo de cada actividad de enseñanza y aprendizaje virtual

\begin{tabular}{|lr|}
\hline 1. & Dimensiones relativas al diseño y planificación de la actividad \\
\hline$\bullet$ & Los objetivos de aprendizaje \\
uso & Las herramientas y materiales informáticos necesarios y sus potencialidades de \\
$\bullet$ & Las posibilidades de agrupamiento de los participantes \\
alumnos. & Las decisiones relacionadas con el tipo de roles que deben ejercer profesores y \\
$\bullet$ & La comunidad de estudiantes a la cual puede ir dirigida la actividad. \\
\hline 2. & Dimensiones relativas a su implementación y desarrollo \\
\hline$\bullet$ & Las fases que pueden establecerse. \\
\hline & Rasgos que caracterizan cada fase. \\
\hline
\end{tabular}


Como se podrá apreciar, apuntamos algunos rasgos de cada actividad entendiendo que cada una de ellas requiere una mayor reflexión por parte del profesor para llevarla a la práctica, sobre todo en aquellos casos que, por la propia naturaleza de la actividad, su desarrollo puede ser complejo. Las actividades que desarrollaremos seguidamente son:

\subsection{Las comunidades virtuales de aprendizaje}

El principal objetivo de las comunidades virtuales de aprendizaje consiste en la creación, desarrollo y mantenimiento de un grupo virtual de estudiantes que tiene como finalidad la construcción de conocimientos de forma compartida mediante la interacción telemática entre todos sus miembros, que en nuestro caso serán alumnos de las aulas (Williams et al., 2000). Por su propia configuración las comunidades virtuales de aprendizaje suelen ser "descentralizadas", es decir, que las características que definen las actividades de aprendizaje que se llevan a cabo no emana en todos los casos de un profesor que las proyecta y dirige, sino más bien son propuestas por los propios alumnos, a veces de forma espontánea y con una duración corta y otras veces de forma muy organizada y de mayor duración.

Las principales tecnologías informáticas que se requieren para la realización de dicha actividad pueden ser, naturalmente además de conexión a Internet, cualquier herramienta de gestión del correo electrónico que pueda administrar las denominadas listas de distribución. Si se desea utilizar herramientas con un desarrollo tecnológico superior se deberá recurrir a los entornos telemáticos colaborativos, que en síntesis consisten en espacios diseñados y creados mediante la tecnología informática y en donde tanto el profesor como los estudiantes pueden enviar mensajes electrónicos que podrán ser vistos por todos los miembros.

En condiciones habituales, no existe la necesidad de realizar agrupamientos de alumnos menores a la totalidad de miembros de la comunidad. Sin embargo, en algunas ocasiones puede ser necesario agrupar temporalmente a varios estudiantes, sobre todo en casos que haya diferencia de intereses temáticos 0 diferencias de expertitud en un campo determinado de conocimiento.

En este tipo de actividad el profesor deja su rol tradicional de instructor y se convierte en un moderador, con roles de regulación de la interacción virtual de la comunidad ejercidas por ejemplo en la admisión o exclusión de alumnos o en establecer las normas de las participaciones de los miembros. En cambio, son los alumnos los que deben tener la iniciativa en todo lo concerniente a las actividades de aprendizaje, con aspectos como la propuesta de una actividad, la participación en la misma o aspectos por ejemplo de ritmos de ejecución (Barberà Badia y Mominó, 2001).

Pueden crearse y desarrollarse comunidades de aprendizaje en las cuales los miembros sean de una misma clase, de diferentes clases de un mismo nivel, de diferentes clases de diferentes niveles o incluso de todo el centro escolar. Sin embargo, las más interesantes pueden ser las que se desarrollen entre alumnos de diferentes centros, o incluso de diferentes países o culturas, de manera que la comunidad virtual lo es realmente dado que en estos últimos casos la presencia física entre los estudiantes es prácticamente inviable.

Para que una comunidad de aprendizaje se convierta en estable deben desarrollarse suficientes contactos entre sus miembros para que lleguen a conocerse y compartirse sus normas de funcionamiento. Una vez consolidada, puede haber dos tipos de actividades típicas en función de su duración. Las actividades de corta duración habitualmente tienen cuatro fases, que se inician mediante el planteamiento 
de un problema o interrogante, seguidamente se plantean varias respuestas o posibilidades de solución, seguidamente se discute cual es la más ajustada a lo que se ha planteado y, en algunos casos, posteriormente a que el que la planteó haya llevado a la práctica su resolución, se vuelve a participar explicando dicha aplicación y los resultados obtenidos. En el segundo caso, en las actividades de larga duración, las fases pueden variar en función del tipo de actividad que se plantee y de su complejidad.

En síntesis, las comunidades virtuales de aprendizaje pueden ser útiles en aquellos casos que se desee promover la integración del estudiante en la cultura y en la sociedad actualmente muy vinculadas a la información y la comunicación, así como en algunos casos para complementar algunas actividades curriculares de enseñanza y aprendizaje relacionados con contenidos culturales. Sin embargo, dada su naturaleza necesariamente no centralizada y basada en la iniciativa del profesor, pueden ser muy desaconsejables si se desea utilizar este tipo de actividades para fomentar el aprendizaje de contenidos curriculares fundamentales (Hewitt, Brett y Scardamalia, 1995).

\subsection{El aprendizaje basado en el uso autónomo de recursos digitales telemáticos}

Este tipo de actividad de enseñanza y aprendizaje virtual tiene como objetivo potenciar la actividad autónoma del estudiante poniendo a su disposición un conjunto de recursos de diferentes tipos con funciones educativas diferentes (Hannafin, Hill y McCarthy, 2000) que deben serle útiles para su aprendizaje.

Para llevar a cabo dicha actividad los estudiantes deben disponer necesariamente de un conjunto bien definido, indexado, accesible y organizado de recursos y materiales en formato digital. Puede haber recursos de muy diferentes tipos, que pueden variar desde estructuras muy simples compuestas de pocos documentos hipertextualizados que pueden ser elaborados con un procesador de textos de uso común hasta "entornos de recursos digitales". Si no se dispone de conexión a Internet, los recursos pueden ser offline, siendo los más comunes materiales preparados por el mismo profesor con contenidos multimedia (audio, imágenes estáticas y dinámicas, representaciones de información...), otros materiales como enciclopedias digitales habitualmente en formato CD-ROM o programas de aprendizaje por medio del ordenador con contenidos específicos. En el caso que se disponga de conexión a Internet, se pueden usar determinadas páginas de Internet previamente predeterminadas o también el uso buscadores de información. En cualquier caso, se debe procurar que haya una cantidad de información muy ajustada a las necesidades de las características de las tareas que se propongan, si es necesario pudiéndose estructurar en lo que Hannafin, Land y Oliver han denominado los entornos de aprendizaje abiertos (2000).

El aprendizaje basado en el uso autónomo de recursos digitales telemáticos se concibe como una actividad en la cual se puede constatar hasta que punto el estudiante es capaz de aprender de forma autónoma y, además, permite valorar hasta qué punto el estudiante es capaz de comprometerse e implicarse de manera responsable en su propio trabajo asumiendo retos de aprendizaje. Por este motivo, esta actividad suele hacerse de forma individual. Sin embargo, en algunas ocasiones puede ser necesario también valorar algunos aspectos de capacidad colaborativa del propio sujeto con otros estudiantes. En este caso se suelen hacer agrupamientos pero no más de parejas o tríos de estudiantes.

Los profesores pueden tener dos formas de intervención muy diferentes, que permiten muchas variaciones en la propia dinámica de la actividad. El aspecto que habitualmente queda enteramente reservado siempre para el profesor es la concreción de los objetivos de la actividad y la evaluación de hasta 
que punto se han conseguido los objetivos. Más allá de este aspecto, quedan otros que deben concretarse como por ejemplo las características de la propia actividad, los materiales disponibles, los ritmos e itinerarios de aprendizaje o los tipos de agrupamiento. Dentro de una gradación de planteamientos diferentes de esta actividad situamos en un extremo de máximo control el caso que sea el profesor quien fije las anteriores características, y en un grado de máxima libertad que sea el estudiante quien pueda decidir qué tareas concretas realiza y en qué orden, qué materiales consulta, qué ritmo de aprendizaje sigue y con quien se agrupa en caso que lo considere necesario. Esta segunda opción puede ser la más adecuada si la actividad se plantea como el análisis de un caso o la resolución de un problema complejo.

El aprendizaje basado en el uso autónomo de recursos digitales telemáticos puede ser muy útil si es desarrollada desde diferentes asignaturas y con contenidos curriculares básicos. Sin embargo, puede provocar ciertos problemas resolubles en el caso que se desee incluir alumnos de diferentes niveles o centros. Aún así, como se puede plantear sin que haya necesidad de que el estudiante se relacione necesariamente con sus compañeros, puede ser muy adecuada si se sitúa dentro de una misma aula y dirigida a todos sus alumnos.

Habitualmente, para el profesor, esta actividad de aprendizaje se desarrolla siguiendo las tres fases típicas, que consisten en presentar la actividad y asegurarse que se comparten sus objetivos y se ha comprendido las condiciones de desarrollo, proporcionar ayudas formativas a los estudiantes cuando están llevándola a cabo y valorar hasta qué punto se han conseguido los objetivos de aprendizaje fijados.

Para finalizar, debe tenerse en cuenta que esta actividad puede ser adecuada siempre y cuando se desee valorar hasta qué punto los estudiantes son capaces de realizar algún trabajo o resolución de problemas de forma autónoma. Por ello, deberán situarse al final de una o varias secuencias didácticas en las cuales el estudiante haya podido construir suficiente conocimiento de todo tipo (tanto declarativo como procedimental y estratégico, y tanto del área curricular como del funcionamiento de estas tecnologías de la información y la comunicación necesarias) para que realmente la exigencia que el profesor le plantee esté dentro de sus potencialidades reales de ejecución.

\subsection{La búsqueda de información en Internet}

Esta actividad persigue el desarrollo de habilidades de exploración de información por parte del estudiante en Internet, por lo que será necesario que anteriormente éste construya conocimiento tanto del contenido del área curricular como de los programas que existen para llevar a cabo este cometido. Así pues, esta actividad puede ser utilizada para dos finalidades complementarias: como objeto propio de aprendizaje o, en el momento que el estudiante ya tiene un cierto dominio de esta habilidad, como una actividad específica que forma parte de una actividad más amplia y compleja, como por ejemplo el aprendizaje basado en el uso autónomo de recursos digitales telemáticos (McFarlane, 2001).

Las principales herramientas que el estudiante va a tener que utilizar son los buscadores y metabuscadores disponibles en Internet, además de ciertas páginas de Internet que tienen la función de reunir diferentes fuentes primarias y secundarias de información directamente relacionada con el tema requerido. Sin embargo, para llevar a cabo una búsqueda y selección adecuada de información no es suficiente con el conocimiento del funcionamiento de dichos programas. Es necesario que desarrolle otras habilidades relacionadas con la validación de los documentos. 
Aunque esta actividad puede realizarse individualmente, resulta muy conveniente que se realice con agrupaciones de alumnos para conseguir diferentes finalidades, como por ejemplo que puedan disponer de ayuda del compañero cuando están desarrollando las habilidades anteriormente citadas, para complementar su búsqueda comparando posteriormente la información encontrada, para seleccionar la más ajustada caso de tratarse de un mismo tema o para cooperar si anteriormente se han repartido la búsqueda de temas complementarios.

Como orientación general, resulta imprescindible que el profesor enseñe a sus estudiantes el uso de varios buscadores que haya seleccionado anteriormente, pues la profusión actual de buscadores en Internet hace que los estudiantes, en este primer paso, puedan sentirse desorientados si se les encomienda a ellos dicha tarea. Posteriormente, es imprescindible que el profesor enseñe el uso estratégico (no sólo técnico) de la utilización de dichos buscadores, realizando el mismo varias búsquedas y explicitando la toma de decisiones que hace, como experto, en relación a la elección de palabras clave y a la selección de documentos. Sólo en casos de alumnos avanzados o que tengan una experiencia suficiente en el uso de estas herramientas es posible dejarles libertad para que ellos mismos elijan los buscadores que deseen.

Aunque podría ser una actividad de aprendizaje que se pueda compartir con otros alumnos de otros centros si es utilizada dentro de otras actividades más amplias, se aconseja que el proceso de enseñanza y aprendizaje de dichas habilidades de búsqueda de información en Internet se realicen dentro de un mismo grupo clase de un nivel educativo determinado.

Podemos destacar tres fases típicas para enseñar estas habilidades a los alumnos. En primer lugar, debe aprender a identificar qué tipo de palabras clave pueden ser importantes para su búsqueda, tanto las directamente relacionadas con el tema como las que forman parte de su campo semántico más próximo. En segundo lugar, debe saber reconocer qué tipo de documentos pueden ser útiles para el trabajo que debe realizar. La valoración de dichos documentos por parte del alumno le va a exigir saber valorar la fiabilidad de la fuente, la validez de su contenido y su adecuación con el nivel de los conocimientos previos que el propio alumno dispone. $\mathrm{Y}$ en tercer lugar va a necesitar ciertas habilidades para organizar la información digital obtenida.

Como nota final, el profesor deberá ser muy cuidadoso en el uso de esta actividad de enseñanza y aprendizaje, puesto que si bien es innegable que Internet puede poner a disposición de los estudiantes una cantidad ingente de información, no es menos cierto que éstos deben disponer de suficientes habilidades para separar la información válida, fiable y útil de la que no lo es tanto para no sentirse "infoxicado".

\subsection{Las discusiones virtuales}

La principal finalidad de la actividad de discusión virtual entre los estudiantes es la construcción compartida de conocimiento sobre temas que son opinables, que carecen de una estructuración formalizada de su contenido o que pueden ser vistos desde diferentes puntos de vista, como por ejemplo en este último caso aquellos contenidos más relacionados con las actitudes, valores y normas.

Como sucedía con la actividad de las comunidades de aprendizaje, para llevar a cabo una discusión virtual es necesario disponer de herramientas de correo electrónico o, si es posible, de entornos colaborativos telemáticos específicos, conocidos comúnmente como debates. Progresivamente estos espacios virtuales de debate van adquiriendo ciertas potencialidades tecnológicas que son muy adecuadas para el desarrollo adecuado de una discusión virtual. Nos estamos refiriendo a la posibilidad de organizar 
gráficamente los mensajes de los estudiantes, o incluso sus contenidos, de manera que quede evidente aspectos de una discusión virtual que son típicos, como por ejemplo el planteamiento del tema, las posturas que se toman ante el tema, los argumentos o contraargumentos que se esgrimen o las conclusiones a las cuales se llega (Barberà Badia y Mominó, 2001).

Aunque puede haber variedades importantes de agrupamientos según sea el tipo de discusión virtual que se plantee, en líneas generales puede ser muy adecuado que se agrupe a los estudiantes en subgrupos de 6-10 estudiantes, utilizando como criterios el interés que pueda mostrar un estudiante por uno de los posibles temas presentados para discutir o, también la posibilidad de agrupar estudiantes con creencias dispares para que el debate sea dinámico e interesante.

Las posibilidades de ejercer y combinar el desarrollo de diferentes roles por parte del profesor y los estudiantes provoca que pueda haber concreciones muy diferentes según sea la aplicación concreta. En cualquier caso, se debe tomar decisiones sobre como mínimo los siguientes aspectos: el tema o temas a discutir, la organización de una o varias discusiones, la asignación de los estudiantes a una o varias discusiones, el modo de iniciar el debate (con una pregunta, un caso real extraído de un periódico, unos datos estadísticos...), el establecimiento, inicio y cierre de las fases y la regulación de la participación del debate.

Aunque un debate virtual realizado entre los alumnos de una misma clase de forma paralela a la enseñanza presencial puede ser muy sugerente, no lo es menos la posibilidad de abrir debates en donde participen alumnos de otras clases, sean del mismo centro educativo o de otros, e incluso cabe la posibilidad de ponerse de acuerdo entre alumnos de lugares del mundo muy distantes entre si.

Aunque no existe para todos los casos unas fases rígidas de obligado seguimiento, en líneas generales la mayoría de los debates virtuales suelen desarrollarse en cinco etapas consecutivas. La primera consiste en la construcción de conocimiento por parte del estudiante de un tema determinado, pudiéndose utilizar la actividad anterior que hemos denominado "búsqueda de información por Internet". En segundo lugar se inicia el debate y puede ser aconsejable que cada alumno envíe un mensaje detallando la postura que toma ante el tema o problema planteado y argumentando el porqué. Aunque en esta segunda fase en ocasiones ya se envían contraargumentos a otras posturas, esto sería lo propio de la tercera fase. En la cuarta fase, que posiblemente puede ser la más extensa temporalmente, los estudiantes deben indagar diferentes alternativas o vías de argumentación intentando no perder el "hilo de la conversación virtual". Por último, como quinta fase puede ser recomendable que cada estudiante, individualmente, realice algún trabajo en el cual se le pida activar habilidades como el análisis, la síntesis o la inferencia a partir de los contenidos de la discusión.

En resumen, los debates virtuales pueden ser una actividad que promueva la construcción adecuada de conocimiento de los alumnos siempre y cuando no se convierta en una mera exposición por parte de cada uno de ellos de su punto de vista sin que haya realmente un intercambio y confrontación de ideas que provoque un cambio significativo en su conocimiento.

\subsection{El trabajo cooperativo virtual}

La actividad basada en el aprendizaje en grupo cooperativo tiene como objetivo principal el desarrollo de una tarea de enseñanza y aprendizaje que únicamente puede ser llevada a cabo mediante la colaboración de todos participantes, que se convierten en miembros activos de un grupo de trabajo. Para 
que se pueda caracterizar el trabajo de un grupo como cooperativo virtual debe cumplir tres requisitos: a) que el objetivo propuesto por el profesor vaya dirigido al grupo y no a sus miembros tomados individualmente, de manera que debe ser conseguido mediante la cooperación entre todos, b) que exista una organización de roles y tareas entre los miembros del grupo de manera que no haya ningún miembro que quede excluido $y, c$ ) que el grupo cooperativo pueda disponer de todo lo necesario (todo tipo de recursos: de contenidos y de instrumentos de comunicación telemática) para su progreso tanto a nivel de interdependencia positiva entre sus miembros como de la propia realización de la tarea (Barberà Badia y Mominó, 2001).

Para que el grupo cooperativo pueda ser operativo a nivel telemático debe poder disponer de alguna herramienta de comunicación virtual que cumpla al menos dos características: que pueda ser útil para desarrollar los procesos interactivos necesarios entre los miembros y que sea fácil compartir documentos. Ambas necesidades se pueden cubrir mediante el uso de programas informáticos de gestión de correo electrónico que permitan establecer una lista de correo compartida. Sin embargo, el proceso puede verse facilitado de manera importante si el grupo puede disponer de un microentorno colaborativo virtual que disponga de las funciones necesarias para cubrir las necesidades de desarrollo de esta actividad. En este caso, y siempre que todas las interacciones entre los miembros se produzcan dentro de este entorno, el profesor va a poder disponer de un muy buen medio para recoger información del proceso cooperativo que siguen los estudiantes y así poder valorar su progreso.

El desarrollo de grupos cooperativos puede hacerse mediante agrupamientos tanto de estudiantes que pertenecen a una misma clase hasta estudiantes que no se puedan ver presencialmente. En el primer caso, la cooperación virtual puede ser un medio complementario a la presencialidad mientras que en el segundo caso toda la interacción cooperativa se producirá virtualmente. En este segundo caso el proceso inicial de creación de interdependencia positiva entre los miembros requerirá más tiempo. Otro aspecto a tener en cuenta en relación al agrupamiento son los criterios que se siguen para llevarlo a cabo. En caso de tratarse de un grupo cooperativo virtual con una tarea estructurada y extensa temporalmente, resulta muy aconsejable que el grupo no supere los 6 miembros y que se asignen a cada grupo de manera que las habilidades que requiera la actividad puedan cubrirse mediante "la suma" o combinación de las habilidades de todos los miembros para que los estudiantes puedan complementarse en su trabajo.

El profesor debe intervenir en todo el proceso de desarrollo del grupo cooperativo, entre otras con funciones como asignar a los estudiantes, expresar y negociar el trabajo a realizar, promover la interdependencia positiva entre los miembros, proponer los roles que se deben asumir en el grupo, proporcionar los materiales necesarios para trabajar (o, si es el caso, los accesos a la información) e intervenir durante el proceso para garantizar que realmente se está llevando a cabo un trabajo cooperativo real. Por su parte, el estudiante debe desarrollar su trabajo en función de la actividad cooperativa que se esté llevando a cabo. Según las características de dicha actividad el estudiante puede asumir roles de tutor o aprendiz (en el caso de la tutoría virtual entre iguales), roles relacionados con una tarea compleja como por ejemplo leer de forma comprensiva (en el caso de la enseñanza recíproca virtual), roles de experto en una parte o dimensión de un contenido (en el caso del grupo cooperativo virtual estrella) o roles vinculados con personas que tienen diferentes puntos de vista sobre un tema social complejo (como sería el caso de desarrollar una representación o "role playing virtual"). 
Para que un grupo cooperativo virtual pueda desarrollarse con éxito y favorezca realmente el aprendizaje de los estudiantes debe estar estructurado en fases en cada una de las cuales se especifique claramente su objetivo, los materiales y contenidos que van a tener que consultarse, las formas de participación de los estudiantes, así como sus roles, el tipo de actividad específica que se debe desarrollar y el periodo temporal en forma de cronograma. No podemos anticipar qué fases son las prototípicas en general ya que cada tipo específico de actividad cooperativa virtual va a tener sus fases características.

\subsection{La elaboración de trabajos hipertextuales}

El planteamiento por parte del profesor de esta actividad puede servir para conseguir dos objetivos diferentes de aprendizaje. En primer lugar, puede desarrollarse como la parte práctica de una secuencia didáctica presencial o virtual en donde se pretenda enseñar a los estudiantes los contenidos conceptuales y procedimentales necesarios para la elaboración de este tipo de materiales. En segundo lugar, y habiéndose conseguido suficientemente este primer objetivo, puede ser útil para valorar hasta qué punto el estudiante 0 estudiantes han construido conocimiento sobre el tema que se plantea y que se pone de manifiesto en los aspectos que caracterizan el propio documento hipertextual (Rouet, 1998).

A menos que esta actividad se diseñe específicamente para conseguir objetivos directamente vinculados con aspectos estéticos, el profesor debe tener muy claro que en líneas generales los criterios que deber servir para valorar el aprendizaje deben tener presente por una parte aquellas propiedades vinculadas a la calidad del contenido complementadas con aquellos aspectos que aporta el hipertexto como valor añadido, como por ejemplo entre otros una estructura global adecuada del conjunto de documentos, la selección apropiada de los conceptos que sirven de enlaces a información relacionada o la calidad o ajuste al tema de los enlaces que se seleccionan de Internet (Nicaise y Crane, 1999).

En función del tipo de documentos hipertextuales que se deseen elaborar se necesitarán hasta tres tipos diferentes de tecnologías informáticas. Si de lo que se trata es de un documento realizado individualmente y con enlaces o vínculos a otros documentos que el propio estudiante ha elaborado bastará con la utilización de algún tipo de programa informático que tenga funciones de inserción de hipervínculos. Ahora bien, si lo que se desea es que el documento también pueda incorporar enlaces a documentos situados en Internet, naturalmente se deberá contar con dicha conexión tanto en el momento de la elaboración del documento como en el momento de revisarlo por parte del profesor. Por último, si se desea que el documento sea elaborado de forma cooperativa por varios alumnos, se necesitará algún tipo de entorno comunicativo que permita la comunicación telemática entre los miembros del grupo y además que admita funciones de compartir documentos.

Como acabamos de apuntar anteriormente, este tipo de actividad puede pensarse tanto para su elaboración individual como en pequeño grupo cooperativo virtual. Ahora bien, también puede diseñarse para que se desarrolle básicamente en el ámbito individual pero que en ciertos momentos se potencie tanto el intercambio de información como el uso puntual de la cooperación entre los estudiantes. Un ejemplo del primer tipo puede ser que los estudiantes envíen a sus compañeros una parte del documento en el momento que está elaborándose para valorar cómo se está enfocando. Un ejemplo del segundo tipo podría ser que los estudiantes cooperen en el momento de buscar información en Internet sobre ciertos puntos relacionados directamente con el tema de su trabajo. 
Como sucedía con la actividad basada en el uso autónomo de los recursos digitales telemáticos, el profesor puede tener diferentes formas de intervención en función de muchos factores pero siempre dentro de un rol alejado del "transmisor de contenido" y próximo a ser considerado como "constructor de contextos educativos virtuales" dentro de los cuales intervenga proporcionando ayudas dirigidas a resolver diferentes necesidades de aprendizaje del estudiante. Naturalmente, además de proporcionar indicaciones sobre las características de la actividad que desee fijar (objetivos, tema, fases de la actividad, criterios de evaluación...) el profesor debe prepararse para poder proveer ayudas e información complementaria a los estudiantes de al menos cuatro tipos: referidas al uso de las propias herramientas o programas informáticos, referidas a la actividad, referidas al contenido o tema y referidas a las estrategias de aprendizaje que deben activarse en cada momento según se determine el trabajo (que pueden estar vinculadas, por ejemplo, a la búsqueda, a la organización y a la presentación de información). Por parte del estudiante, en este tipo de actividades debe actuar con un alto grado de motivación, iniciativa y autonomía, aprendiendo a tomar decisiones y a autorregular todo el proceso de elaboración del documento hipertextual.

Aunque esta actividad puede ser realizada de forma individual, puede resultar muy interesante enfocarla como una actividad colaborativa virtual o aún más como una actividad auténtica y significativa con características próximas a los contextos reales. En el primer caso, al consistir en un documento elaborado cooperativamente, pueden participar desde alumnos que realicen el trabajo presencialmente pero compartan dicho documento mediante alguna herramienta de comunicación telemática o podría ser incluso que la actividad se desarrolle totalmente virtualizada y que puedan participar alumnos de diferentes emplazamientos. Esto último podría ser muy motivante en el caso que el documento que se desarrolle sea, por ejemplo, una página de Internet.

Aunque resulta difícil establecer algunas fases que puedan abarcar a la totalidad de posibilidades bajo las cuales se puede plantear dicha actividad, si que nos aventuramos a distinguir tres fases que podrían seguir una amplia variedad de concreciones. Así pues, situamos en primer lugar un periodo de planteamiento de la actividad, en el cual el profesor y los estudiantes "negocian" las características que debe tener el documento así como las condiciones bajo las cuales se debe realizar la actividad. A esta primera parte le seguiría un segundo periodo que sería propiamente la elaboración del documento por parte del estudiante, y en la cual el profesor debería seguir muy de cerca el progreso de confección de cada uno de los documentos. Por último, se podría diseñar una tercera fase, que podría denominarse de difusión, y en la cual los estudiantes ponen a la luz el documento para que pueda ser observado, y si es necesario examinado, por los demás. Esta tercera parte puede realizarse ya dentro del propio grupo de estudiantes, en un espacio virtual compartido, o si se trata de una página de Internet puesta a disposición de todos los internautas.

En síntesis, se trata de una actividad que puede resultar muy estimulante y fructífera para los alumnos sobre todo si se huye de desarrollos en donde lo que parezca que únicamente cuente son aspectos estéticos y de despliegue tecnológico $y$, en cambio, se potencien realmente las estrategias de aprendizaje de los alumnos relacionadas con la búsqueda, la selección y la organización de la información, entre otras. 


\subsection{Las actividades de autoevaluación}

Las actividades de autoevaluación tienen como principal objetivo el proporcionar a los estudiantes información tanto del proceso de aprendizaje que están siguiendo como de la calidad del conocimiento que están construyendo siempre teniendo en cuenta que dicha información debe serles útil para tomar decisiones para, si resulta conveniente, reorientar su proceso de aprendizaje en el sentido que sea necesario, tanto para aspectos conceptuales, procedimentales, estratégicos o metacognitivos. Por ello, debe considerarse como una actividad que, en cierto sentido, se pone a disposición o se sitúa dentro de otra para favorecer el proceso de aprendizaje del alumno.

Puede haber diferentes tipos de herramientas informáticas para llevar a cabo estas actividades de autoevaluación, de entre los cuales destacamos los instrumentos de corrección automática virtual (Olea y Ponsoda, 1998) y la corrección cualitativa virtual. Los primeros suelen tener una base tecnológica que permite plantear, por citar algunos ejemplos, preguntas de elección múltiple con una o varias respuestas correctas, preguntas en las cuales la respuesta debe ser pocas palabras concretas o el establecimiento de relaciones entre los elementos de dos columnas de información. En la mayoría de los casos, se dirigen a la autoevaluación de conocimientos de hechos, conceptos o relaciones conceptuales (a veces también pueden ser respuestas concretas a ejercicios) en las cuales la respuesta puede variar en su complejidad, desde valorar la respuesta del alumno hasta utilizar frases que expliquen el porqué de la corrección de la respuesta 0 , en caso de error, que recomienden nuevos procesos de aprendizaje para mejorar los resultados. El segundo tipo de instrumentos de corrección cualitativa virtual suelen ser más complejos y pueden consistir en proporcionar a los estudiantes criterios de evaluación formativa que les permita valorar hasta qué punto han conseguido el objetivo que se proponía en alguna actividad o también pueden proporcionar información sobre el proceso de aprendizaje seguido para conseguir dichos objetivos.

A pesar de que habitualmente este tipo de actividades están dirigidas a cada estudiante, podrían también utilizarse para proporcionar virtualmente información tanto conceptual como procedimental o estratégica en relación al trabajo realizado por un grupo colaborativo. En ninguno de los dos casos se requeriría un tipo específico de agrupamiento de los estudiantes.

La principal ocupación del profesor para desarrollar este tipo de actividades consiste en decidir que ayudas de este tipo va a requerir el estudiante, elaborar cada una de las respuestas y decidir de qué modo las pone a disposición del estudiante. Por parte de los estudiantes, deben ser capaces de utilizarlas en cada momento en función del proceso de aprendizaje que estén siguiendo.

Este tipo de actividad puede ser utilizable tanto presencial como virtualmente. En este segundo caso, la corrección automática virtual puede ponerse a disposición de un número elevado de alumnos, mientras que la corrección cualitativa virtual no es tan estandarizable, dado que siempre se debe concretar para el proceso de enseñanza y aprendizaje específico en el cual se desarrolle la evaluación de los objetivos de aprendizaje.

Durante la implementación de esta actividad, para el profesor la corrección automática virtual no debe requerirle ningún tipo de intervención específica a menos que alguna de las tareas diseñadas presente problemas. En cambio, para poder proporcionar a los estudiantes actividades ajustadas de corrección cualitativa virtual, el profesor debe estar muy atento al proceso específico que se está siguiendo y, a partir 
de un diagnóstico de dicho proceso, ser capaz de proporcionar información evaluativa muy contextualizada y ajustada al proceso concreto que el estudiante o estudiantes están siguiendo.

En síntesis, las actividades de autoevaluación pueden ser muy útiles para los estudiantes siempre y cuando les proporcionen información adecuada para que puedan llegar a cotas superiores de autonomía en su aprendizaje y de comprensión de los contenidos.

\subsection{La confección de bases de datos}

La actividad de confección de bases de datos puede ser utilizada por el profesor para potenciar las habilidades de los estudiantes vinculadas con la búsqueda, clasificación e interrogación de datos, que pueden provenir de diferentes fuentes (como por ejemplo, de Internet) y que pueden ser de diferente naturaleza y de la mayoría de áreas curriculares. En este sentido, puede ser utilizada tanto para favorecer el desarrollo de dichas habilidades como también para ser utilizada en actividades más complejas como por ejemplo poder disponer de información organizada para el trabajo cooperativo virtual o para el trabajo mediante proyectos electrónicos.

Las herramientas informáticas necesarias están en función de las variables que influyen en la concreción de la actividad, de entre las cuales destacamos el tipo y fuente de información, los instrumentos informáticos de registro de datos y algún tipo de herramienta de comunicación telemática que permita acceder al fichero compartido de los datos. En el caso de proponer una actividad compleja, los estudiantes deberán tener acceso a Internet, entre otras fuentes, y deberán disponer de programas informáticos que tengan funciones de clasificación de información, y que no siempre deben ser necesariamente programas de bases de dados, puesto que en casos de información reducida se podrían utilizar con esta misma finalidad los procesadores de texto o las hojas de cálculo.

Aunque este tipo de actividades pueden proponerse individualmente, resulta sumamente interesante que se diseñen y planteen como actividad de trabajo en grupo, dentro del cual cada miembro puede ejercer diferentes responsabilidades. En este caso, el agrupamiento puede hacerse en función de las posibles responsabilidades que se puedan concretar, y las cuales podrían surgir de las habilidades que los estudiantes deben poner en juego en esta actividad.

Si nos centramos en esta segunda posibilidad, de plantear la actividad organizada en grupos de trabajo, el profesor debe situarse, como ocurría con otras actividades, como "creador de contextos educativos virtuales", interviniendo en los momentos que crea necesario, sobre todo en un primer momento de plantear la actividad, constituir los grupos y asignar roles, durante el seguimiento de dicha actividad y al final en el momento de valorar el grado de consecución de los objetivos. Por otra parte el estudiante debe ejercer los roles típicos dentro de un trabajo en grupo, asumiendo en cada caso el rol que le corresponde, tanto relacionado con el propio trabajo como en los roles típicos de interacción social que exige un trabajo compartido.

Esta actividad puede plantearse de forma flexible para que en si misma no produzca ningún problema en el caso que se dirija a estudiantes que no puedan interactuar de forma presencial. Siempre que la tecnología lo permita, puede ser perfectamente factible que se realice contando con que los miembros que van a colaborar sean de centros diferentes o incluso de países o culturas diferentes. 
Si nos situamos en el desarrollo de la actividad, puede haber variaciones importantes en su organización que afecten al establecimiento de fases diferentes. Sin embargo, y teniendo en cuenta que los estudiantes ya poseen suficiente conocimiento de los aspectos técnicos de los programas informáticos, cualquier profesor que quiera implementarla deberá tener en cuenta los momentos, no necesariamente presentados de forma secuenciada. En primer lugar, los estudiantes deberán poseer suficiente conocimiento del tema de referencia de la base de datos, al menos para poder establecer con suficiente criterio los campos de la base de datos. En segundo lugar, los estudiantes deberán tomar decisiones sobre los campos que formaran parte de dicho documento. Aunque esta tarea puede ser relativamente fácil en algunos casos en los cuales ya existen protocolos para hacerlo (como por ejemplo, una base de datos bibliográfica) esta tarea puede no resultar tan fácil en el caso que los registros de la base de datos tengan una estructura jerarquizada (como puede suceder, por ejemplo, en casos en donde la estructura de la base de datos tenga una organización de, por ejemplo, un mapa de conceptos). En tercer lugar, se debe iniciar la búsqueda de información para ir acumulando campos de dicha base de datos. En este caso, se deberá tener presente la necesidad de una revisión periódica de los campos que se han establecido. Posiblemente la última fase (aunque esto puede depender de si esta actividad está integrada en otra de mayor duración) deba consistir en la interrogación de dicha base de datos con el objetivo que los filtros que se establezcan puedan proporcionar en cada caso información relevante para poder responder a preguntas que le podamos "formular".

\subsection{El trabajo mediante proyectos electrónicos}

Los proyectos electrónicos son actividades complejas en las cuales el estudiante debe poner en juego de forma adecuada una gran variedad de habilidades que pongan de manifiesto que ha conseguido los objetivos que se le proponían. Aunque puede concretarse de formas muy variadas, en todos los casos esta actividad posee tres componentes: a) los estudiantes generan una cuestión o problema que sirve para organizar y guiar las necesidades de aprendizaje, b) dichos estudiantes elaboran y presentan un producto final dirigido a dar respuesta a la cuestión o problema planteado, y c) la actividad se desarrolla con la ayuda de un entorno telemático especialmente ideado para dar soporte a las acciones de los estudiantes (Laffey, Tupper, Musser y Wedman, 1998).

Existen entornos telemáticos complejos especialmente diseñados para esta necesidad, que disponen de diferentes herramientas para favorecer la provisión de ayudas de los profesores, la planificación del trabajo de los estudiantes, el acceso a los recursos de información (Internet, bases de datos específicas...), la representación gráfica de conocimiento de los estudiantes, la comunicación y colaboración entre los estudiantes y los procesos evaluativos de reflexión y revisión del proceso de elaboración del proyecto por parte del alumno. Aunque disponer de este tipo de entornos podría ser lo óptimo, esta actividad podría desarrollarse también con herramientas y materiales informáticos relativamente al alcance de las posibilidades de cualquier centro educativo, haciendo un uso orientado de los programas informáticos de ofimática, utilizando el acceso a Internet, utilizando sus buscadores y bases de datos que los propios alumnos hayan podido elaborar previamente y utilizando ajustadamente los instrumentos de comunicación telemática como por ejemplo algún programa de gestión del correo electrónico.

La actividad de proyectos electrónicos puede diseñarse como una actividad individual o en grupo, dependiendo en cada caso de los objetivos que se deseen conseguir. Sin embargo, en ambos casos debe 
pensarse como una actividad en la cual exista un alto nivel de interacción telemática entre los participantes. Incluso en el caso de plantearse como una actividad individual, debe considerarse la posibilidad que pueda existir colaboraciones puntuales entre los alumnos, tanto a nivel de un gran grupo como en el caso que se indiquen momentos en los cuales se debe cooperar como miembros de un grupo pequeño.

Como también sucedía con algunas de las anteriores actividades presentadas, el profesor puede intervenir en todos los momentos de realización de cada proyecto como "coordinador" del proceso. Sin embargo, dada la naturaleza de la actividad, en este caso cobra especial relevancia que su intervención se centre muy específicamente en una gran parte de los casos en proporcionar ayudas para que el o los estudiantes puedan tomar las decisiones más adecuadas para progresar en la realización de sus respectivos proyectos. En consecuencia, se espera de los estudiantes que tomen la iniciativa en la mayoría de los procesos de aprendizaje y que sean activos desde el momento de plantear su pregunta de indagación o problema hasta el final de la actividad.

Las características de esta actividad la hacen aconsejable para ser propuesta especialmente para grupos de estudiantes que se encuentren en diferentes centros educativos sin que para ello deba ser necesaria un contacto presencial siempre y cuando se pueda contar con unos instrumentos informáticos que permitan afrontar el reto con garantías.

La diversidad de recorridos que puede tomar el proceso de elaboración de cada uno de los proyectos hace inviable el establecimiento de fases fijas en su desarrollo más allá que el inicio y el final que hemos indicado. Ahora bien, dentro de este marco temporal es necesario que, siguiendo el orden que sea, el estudiante se fije metas contextuales, divida las metas complejas en objetivos alcanzables, planifique periodos de trabajo para alcanzar dichas metas, planifique y anticipe los recursos disponibles para alcanzar cada objetivo, se comunique con los otros miembros para compartir y discutir problemas, ideas y resultados, pida ayuda cuando se deba enfrentar a problemas para los cuales juzga que no está preparado, diagnosticando problemas, elaborando representaciones gráficas de su conocimiento que faciliten los procesos de encuadre y reestructuración de ideas (Land y Greene, 2000).

\subsection{La producción de presentaciones multimedia}

La utilización de la actividad de enseñanza y aprendizaje virtual basada en la producción de presentaciones multimedia puede ser muy útil para conseguir que los estudiantes desarrollen las habilidades vinculadas con la síntesis y la comunicación de la información, por lo que en muchas ocasiones suele ser una actividad que puede integrarse como tarea final de otra actividad con una duración temporal mayor.

Para llevar a cabo dicha actividad es preciso poder contar al menos con dos tipos de herramientas tecnológicas. Por un lado, se requiere el uso de determinados programas que faciliten la organización sintética de información en documentos en formato digital, siendo quizá el más popular y accesible el programa Microsoft PowerPoint (2000). Por el otro, debe disponerse de un programa de comunicación telemática que permita el envío de documentos y, si la preparación debe realizarse en grupo, se debe contar con algún tipo de entorno colaborativo virtual. Si el destinatario es un único individuo bastará con un programa de gestión de correo electrónico. Sin embargo, suele ser muy corriente que este tipo de actividades de presentación de información vayan dirigidos a una audiencia más amplia con el propósito, en 
muchas ocasiones, de discutir dicha información. En este caso se precisará disponer de algún tipo de entorno colaborativo virtual.

Dado que en muchas ocasiones la producción de presentaciones multimedia puede situarse dentro de una actividad más amplia, la configuración de esta última actividad va a determinar la necesidad de agrupar o no a los alumnos. En otras palabras, si el trabajo ha sido realizado por un grupo de estudiantes, va a resultar en principio lógico que la actividad de presentación de información multimedia se realice siguiendo esta misma organización. Ahora bien, en este tipo de tarea cabe otro tipo de agrupaciones, que se refiere a la audiencia que va a ser la receptora del trabajo. En este punto va a ser necesario que el profesor tome decisiones en este sentido, decidiendo si la audiencia va a ser todo el grupo, una parte de los miembros de la comunidad virtual o incluso otro de los grupos que se hayan formado.

Aunque este tipo de actividad puede plantearse como una tarea que el o los estudiantes deben realizar de forma independiente del profesor, creemos que es necesario que éste no se desentienda en el momento de ofrecer ayudas a los estudiantes en todo el proceso. Este rol es irrenunciable en el caso que la meta del profesor sea precisamente enseñar a los estudiantes a producir una presentación multimedia. En este caso, debe intervenir directamente mediante estrategias instruccionales que ofrezcan a los estudiantes modelos cognitivos de actuación contextual que les permitan aprender a tomar decisiones útiles para esta actividad y, como consecuencia, que desarrollen las habilidades de aprendizaje específicas (como comprender, sintetizar u organizar información, entre otras) para este tipo de tareas de presentación de información.

Las características de esta actividad la hacen especialmente recomendable para ser usada de forma virtual, aunque si es posible la preparación puede hacerse a nivel presencial. Así pues, la demanda puede ir dirigida sin producir excesivas dificultades a una comunidad virtual de estudiantes, que si es preciso podrán prepararla de forma colaborativa.

Tomando como ejemplo una actividad de producción de una presentación multimedia en grupo, desde el punto de vista del alumno una primera fase consiste en delimitar el contenido al cual se va a referenciar en la presentación. En un segundo momento se deberá identificar (básicamente mediante habilidades de comprensión y síntesis) la información que se desea presentar teniendo en cuenta siempre algunos factores como entre otros muchos el objetivo específico que se desea conseguir 0 las características de la audiencia. En un tercer momento debe tomarse decisiones sobre los componentes concretos (texto, audio, gráficos, ilustraciones estáticas o dinámicas, vídeo...) que van a formar parte de la exhibición multimedia y el orden de presentación. En función de una progresiva complejidad en la presentación (un ejemplo complejo puede ser una conferencia presentada virtualmente que contemple todas estas posibilidades) puede ser necesario incluir otras fases como por ejemplo una revisión previa de dicha presentación para corregir errores.

\subsection{Las investigaciones virtuales}

Las investigaciones virtuales son un tipo de actividad de enseñanza y aprendizaje que tienen por objeto promover el desarrollo de los conocimientos de los alumnos y sus habilidades para aprender contenidos principalmente científicos vinculadas a tareas como proponer hipótesis como problemas amplios a resolver y buscar posibles soluciones o respuestas a dichos problemas utilizando la aplicación del método científico con fines didácticos (Krajcik, Soloway, Brumenfeld y Marx, 2000). 
El tipo de herramienta más difundida para promover este tipo de conocimientos en los alumnos son los programas de simulación utilizados con el ordenador. Este tipo de entornos de aprendizaje están especialmente diseñados con diversas herramientas que permitan a los estudiantes realizar actividades de indagación y descubrimiento basadas en el planteamiento de hipótesis, la introducción de información en el simulador y la obtención y observación de los resultados, como por ejemplo puede ocurrir con los circuitos eléctricos creados informáticamente. (Jong y Joolingen, 1998).

En principio este tipo de actividades con el uso de programas de simulación están dirigidas al aprendizaje individual. A pesar de esto, no debe descartarse la posibilidad que este mismo tipo de actividades se lleve a cabo organizada en grupos colaborativos, aunque para ello deban diseñarse entornos específicos colaborativos en donde llevar a cabo la indagación científica, basados en la mayoría de los casos en construir en colaboración virtual un discurso científico común mediante instrumentos de representación compartida de la información (Suthers, Toth y Weiner, 1997; Suthers, 1999).

El profesor debe convertirse en facilitador de la indagación del estudiante, acompañando y guiando su trabajo, y ofreciendo ayudas individuales según las necesidades. Por otro lado, el estudiante debe intentar desarrollar las actividades de forma autónoma, que incluyen las acciones que ya hemos indicado.

Si se propone una utilización individual y presencial de los programas de simulación esta actividad no puede ser considerada como una actividad virtualizable a menos que se disponga de un acceso telemático a dicho programa y que los estudiantes puedan acceder a él desde fuera del aula. Ahora bien, y entendiendo que el aprendizaje puede ser considerado como un proceso progresivo de construcción de significados compartidos dentro de un grupo determinado de personas, la utilización de los entornos de indagación colaborativa conllevan la necesidad de potenciar la necesaria interacción entre los alumnos, por lo que puede ser muy interesante que dicho proceso colaborativo pueda ser llevado a cabo por estudiantes que no necesariamente puedan interactuar presencialmente.

En síntesis, este tipo de tareas pueden posibilitar que los estudiantes aprendan el método científico y los conocimientos de la ciencia mediante una metodología didáctica basada en el descubrimiento que tiene como base la utilización de programas de simulación como los entornos informáticos más difundidos pero que también puede llevarse a cabo mediante procesos colaborativos de construcción compartida de significados.

\section{ORIENTACIONES PARA EL USO EFICIENTE DE UN AULA VIRTUAL}

A modo de conclusión queremos finalizar con algunas indicaciones que pueden ser útiles para el uso concreto y eficiente de un aula virtual. Para facilitar su lectura las hemos dividido en cuatro apartados:

\subsection{Respecto a su arquitectura:}

- La tecnología se debe elegir en relación a las funciones educativas que cumplirá el aula virtual (socializadora, comunicativa, organizadora, etc.).

- Los elementos seleccionados (links, debates,...) deben estar de acuerdo con los objetivos educativos que se persiguen y que deben estar especificados previamente.

- La concepción del aula y su organización general pueden responder a patrones diversos (distribución de la información, síntesis de la información, inclusión en una comunidad,...) que a 
veces se sostienen por decisiones implícitas que es necesario explicitar para actuar sobre ellas eficientemente.

- Las características de acceso al aula virtual se deben desarrollar siguiendo criterios claros para los alumnos (quién accederá, cuándo y desde dónde se puede acceder).

\subsection{Respecto a la interacción producida:}

- Una mayor participación que conlleve interacción con feedback entre profesores y alumnos y entre alumnos mismos es la clave para mantener el sentido del aula virtual.

- Los intercambios comunicativos virtuales tienen que ser frecuentes, ágiles y diversificados virtualmente en base a una actividad de enseñanza y aprendizaje o de evaluación, sean estas virtuales o presenciales.

- Las diferentes características, necesidades e intereses de los alumnos puede quedar reflejada en la diversificación de itinerarios de trabajo que facilita la tecnología.

\subsection{Respecto al papel del profesor en el aula virtual:}

- La docencia mediante aulas virtuales exige tener en cuenta de un modo central aspectos relativos a la planificación de la programación de la actividad en un aula virtual, así como la previsión de su desarrollo.

- El establecer normas y criterios claros y conjuntos resulta fundamental para el seguimiento y la evaluación de la actividad realizada virtualmente por el alumno.

- La concepción mediática de la tecnología facilita el enfoque multidisciplinar e interdisciplinar de los planteamientos docentes.

- La implicación y el apoyo del profesor en las tareas virtuales es imprescindible en el inicio del uso de un aula virtual.

\subsection{Respecto al tipo de actividad desarrollada:}

- El formato de presentación y desarrollo de las actividades formativas que se incluyen en un aula virtual tiene que responder a realidades específicas que la tecnología pueda aportar de modo particular a los procesos educativos (simulaciones, visualización de procesos, etc.).

- La decisión sobre el nivel cognitivo que las actividades de enseñanza y aprendizaje virtual debe incorporar actividades que desarrollen habilidades de alto nivel (argumentar, valorar, anticipar, etc.)

- El planteamiento de actividades de enseñanza y aprendizaje virtual ha de seguir los criterios de la enseñanza adaptativa en tanto que aporten elementos de flexibilización tanto a los alumnos como a los profesores.

\section{REFERENCIAS BIBLIOGRÁFICAS}

BarberÀ, E. (coord.), BAdiA, A. y Mominó, J. M. (2001). La incógnita de la educación a distancia. Barcelona: ICE UB/Horsori. 
Hannafin, M. J., Hill, J. R. y MccaRThy, J. E., (2000). Designing resource-based learning and performance support systems. En D. A. Wiley (ed.), The instructional use of learning objects. Versión disponible online en:

http://reusability.org/read/chapters/hannafin.doc [mayo del 2001].

Hannafin, M., Land, S. y Oliver, K. (2000). Entornos de aprendizaje abiertos: fundamentos, métodos y modelos. En , Ch. M. Reigeluth (Ed.), Diseño de la instrucción. Teorías y modelos, (125-152). Madrid: Santillana Aula XXI.

Hewitt, J., Brett, C. y Scardamalia, M. (1995). Schools for thought: transforming classrooms into learning communities. Symposium 29.57 y Poster Session 31.60. AERA San Francisco. Versión disponible online en:

http://csile.oise.utoronto.ca/abstracts/kn_build/ [abril del 2002].

JONG, T. D. y JOOLONGEN, W. V. (1998). El entorno SMISLE: diseño y aprendizaje con entornos integrados de simulación. En C. Vizcarro y J. A. León, Nuevas tecnologías para el aprendizaje, (53-64). Madrid: Pirámide.

Krajcik, J., Soloway, E., Brumenfeld, P. y Marx, R. (2000). Una andamiaje de herramientas tecnológicas para promover la enseñanza y el aprendizaje de ciencias. En C. Dede (comp.), Aprendiendo con tecnología, (60-77). Buenos Aires: Paidós.

LAfFey, J., Tupper, T., Musser, D. y Wedman, J. (1998). A computer mediated support system for project based learning. Educational Technology Research and Development, 46 (1), págs. 73-86.

Land, S. y Greene, B. (2000). Project-based learning with the World Wide Web: A qualitative study of resource integration. Educational Technology Research and Development, 48 (1), págs. 45-68.

Macfarlane, A. (2001). El aprendizaje y las tecnologías de la información. Madrid: Santillana Aula XXI.

Nicaise, M. y CRANe, M. (1999). Knowledge constructing through hypermedia authoring. Educational Technology Research and Development, 47 (1), págs. 29-50.

OlEA, J. y PONSODA, V. (1998). Evaluación informatizada en contextos de aprendizaje. En C. Vizcarro y J. A. León, Nuevas tecnologías para el aprendizaje, (161-175). Madrid: Pirámide.

PASSEY, D. (2000). Developing teaching strategies for distance (out-of-school) learning in primary and secondary schools. Education Media International, 37 (1),45-57.

ROUET, J. F. (1998). Sistemas de Hipertexto: de los modelos cognitivos a las aplicaciones educativas. En C. Vizcarro y J. A. León, Nuevas tecnologías para el aprendizaje, (87-101). Madrid: Pirámide.

SUTHERS, D. (1999). Representational support for collaborative Inquiry. Proceedings of the $32^{\text {nd }}$ International Conference on Systems Sciences. Hawaii. Págs. 1-14.

SUTHERS, D., Toth, E. y WeINER, A. (1997). An integrated approach to implementing collaborative inquiry in the classroom. Proceedings of the $2^{\text {nd }}$ International Conference of the Computer Supported Collaborative Learning. Toronto. págs. 272-279. 
Williams, S., Burgess, K., Bray, M., Bransford, J., Goldman, S. y el Grupo de Cognición y Tecnología de Vanderbilt (CTGV). (2000). La tecnología y el aprendizaje en las aulas de las escuelas para el pensamiento. En C. Dede (comp.), Aprendiendo con tecnología, (139-165). Buenos Aires: Paidós. 


\title{
Contactar
}

Revista lberoamericana de Educación

\author{
Principal OEI
}

